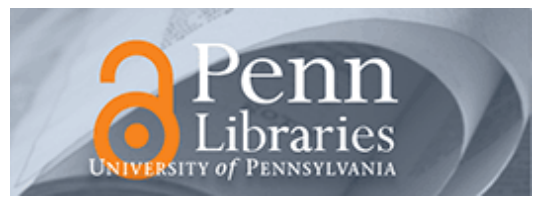

University of Pennsylvania

ScholarlyCommons

Statistics Papers

Wharton Faculty Research

2009

\title{
Path Data in Marketing: An Integrative Framework and Prospectus for Model Building
}

Sam K. Hui

Peter S. Fader

University of Pennsylvania

Eric T. Bradlow

University of Pennsylvania

Follow this and additional works at: https://repository.upenn.edu/statistics_papers

Part of the Applied Statistics Commons, Business Analytics Commons, Management Sciences and Quantitative Methods Commons, Marketing Commons, Statistical Models Commons, and the Statistical Theory Commons

\section{Recommended Citation}

Hui, S. K., Fader, P. S., \& Bradlow, E. T. (2009). Path Data in Marketing: An Integrative Framework and Prospectus for Model Building. Marketing Science, 28 (2), 320-335. http://dx.doi.org/10.1287/ mksc. 1080.0400

This paper is posted at ScholarlyCommons. https://repository.upenn.edu/statistics_papers/657

For more information, please contact repository@pobox.upenn.edu. 


\title{
Path Data in Marketing: An Integrative Framework and Prospectus for Model Building
}

\author{
Abstract \\ Many data sets, from different and seemingly unrelated marketing domains, all involve paths-records of \\ consumers' movements in a spatial configuration. Path data contain valuable information for marketing \\ researchers because they describe how consumers interact with their environment and make dynamic \\ choices. As data collection technologies improve and researchers continue to ask deeper questions \\ about consumers' motivations and behaviors, path data sets will become more common and will play a \\ more central role in marketing research. \\ To guide future research in this area, we review the previous literature, propose a formal definition of a \\ path (in a marketing context), and derive a unifying framework that allows us to classify different kinds of \\ paths. We identify and discuss two primary dimensions (characteristics of the spatial configuration and \\ the agent) as well as six underlying subdimensions. Based on this framework, we cover a range of \\ important operational issues that should be taken into account as researchers begin to build formal \\ models of path-related phenomena. We close with a brief look into the future of path-based models, and a \\ call for researchers to address some of these emerging issues.
}

\section{Keywords}

path data, path models, integrative review, grocery shopping, eye tracking, Web browsing, clickstream, information acceleration

\section{Disciplines}

Applied Statistics | Business | Business Analytics | Management Sciences and Quantitative Methods | Marketing | Statistical Models | Statistical Theory | Statistics and Probability 
Path Data in Marketing:

An Integrative Framework and Prospectus for Model-Building

\author{
Sam K. Hui \\ Peter S. Fader \\ Eric T. Bradlow*
}

$1^{\text {st }}$ Submission: February 6, 2007

Revised: August 7, 2007

* Sam K. Hui is a doctoral candidate in Marketing, Peter S. Fader is The Frances and Pei-Yuan Chia Professor, Professor in Marketing, and Eric T. Bradlow is The K. P. Chao Professor, Professor of Marketing, Statistics, and Education, and Academic Director of The Wharton Small Business Development Center, at the Wharton School of the University of Pennsylvania. Corresponding author: Sam K. Hui (kchui@wharton.upenn.edu). The authors are grateful for the feedback and encouragement from Herb Sorensen of Sorensen Associates. 


\title{
Path Data in Marketing: An Integrative Framework and Prospectus for Model-Building
}

\begin{abstract}
Many datasets, from different and seemingly unrelated marketing domains, all involve “paths”- records of consumers’ movements in a spatial configuration. Path data contain valuable information for marketing researchers because they describe how consumers interact with their environment and make dynamic choices. As data collection technologies improve and researchers continue to ask deeper questions about consumers' motivations and behaviors, path datasets will become more common, and play a more central role in marketing research.

To guide future research in this area, we review the previous literature, propose a formal definition of paths, and derive a unifying framework that allows us to classify different kinds of paths. We identify and discuss two primary dimensions (characteristics of the spatial configuration and the agent) as well as six underlying sub-dimensions. Based on this framework, we cover a range of important operational issues that should be taken into account as researchers begin to build formal models of path-related phenomena. We close with a brief look into the future of path-based models, and a call for researchers to address some of these emerging issues.
\end{abstract}




\section{Introduction}

Consider the following domains of data collection and active research in marketing:

(i) Grocery shopping: A grocery store installs Radio-Frequency Identification (RFID) tags underneath shopping carts to track consumers' in-store movements.

(ii) Eye tracking: Researchers ask subjects to view print advertisements and capture their eye movements using infrared corneal reflection technology.

(iii) Web browsing: Consumers’ web-browsing patterns are tracked by recording the sequence of webpages visited in each session.

(iv) Information acceleration: Researchers immerse subjects in a multi-media environment to understand how they collect information and make decisions about a radically new product or service.

At first thought, these domains may not seem to share much in common: they involve different settings and describe different kinds of behaviors. For instance, while grocery shopping and eye tracking involve actual physical movements, web clickstream and information acceleration occur only in virtual environments. In addition, consumers' underlying goals will generally be quite different in each of these areas. In some cases, the consumer's aim may be to purchase products, while in the other cases the main goal is to gather information. Despite these differences on the surface, a cursory look at Figure 1 suggests that all of these domains share a common theme: they all involve the collection and analysis of different kinds of path data.

[Insert Figure 1 about here]

Path data (which will be defined formally in Section 2) create a record of a person's movement in a spatial configuration. They inform us about how a consumer interacts with his environment to achieve his goal(s). In this paper, we argue that there is a need for a closer focus 
on, and integration of, path-related research in marketing for three reasons. First, the study of paths, in conjunction with other data sources (e.g., surveys, transaction data), may lead to a deeper understanding of consumer behavior; e.g., consumers' paths may be informative about their decision processes and goal orientations. For instance, Montgomery et al. (2004) demonstrated that by taking into account a consumer's page-to-page browsing path, his online purchase behavior can be predicted more accurately (i.e., paths predict choices). Second, understanding the paths consumers follow is, in some cases, the primary dependent variable of interest by itself (Bradlow et al. 2005). For example, understanding store traffic patterns may help retailers optimize their store layout (Vrechopoulos et al. 2004); similarly, studying eye movements may lead to insights on how consumers shift their visual attention and thus inform advertisers on how to design ads to maximize their impact (Fox et al. 1998). Finally, as we demonstrate in Section 3, path data are emerging in many areas of marketing. As data collection technologies continue to improve, we will likely see an explosive growth in path-related research. Thus, it is important for us to understand the underlying dimensions and associated modeling challenges for path data and to take full advantage of these new data opportunities.

Although marketing researchers have (implicitly) collected and studied path data, the "path" aspect of the data is usually under-exploited and the commonalities that are present across domains are rarely recognized and appreciated. What is missing, we believe, is a general framework that organizes and unifies path-related marketing contexts. Such a framework will serve three important research purposes. First, it allows us to categorize different path data to understand the similarities and differences among them; this will be helpful for the researcher who is looking for analogous models and managerial settings. Second, with a unified framework, modelers can identify a set of common issues in the analysis and modeling of paths, so that 
researchers can tackle problems that arise across different areas simultaneously. Third, a formal framework will help researchers borrow the tools developed in other disciplines (e.g., models of animals’ and pedestrians’ movement) to analyze paths in marketing.

The central goal of our paper is to provide such a framework. In the next section, we define the kinds of paths that make up the focus of this paper, and provide a formal definition of a path that is grounded in real analysis (Rudin 1976) and graph theory (Bollobas 1979). We then describe our general framework in detail, focusing on two key components: the spatial configuration and the agent who follows the path of interest. In Section 3, we review the different types of path data that arise in four active areas of marketing research: retail/service environments, advertising studies, e-commerce, and experimental research. We also briefly review the literature outside of marketing to help describe the broader context and framework that we develop. Based on our framework and literature review, we identify modeling issues that will commonly arise for researchers who are attempting to understand or predict path behavior. Finally, we conclude by offering some observations and recommendations for future research in this area.

\section{A Framework for Path Data}

In this paper, we focus on paths that correspond to

Definition: a conscious (Blackmore 2003) agent's movement in a physical or simulated (Kalawsky 1993) environment that is observable. ${ }^{1}$

A path can be denoted by a three-tuple $P=\left\{S, A, X_{A}(t)\right\}$. $S$ denotes an observable, physical or simulated (e.g., web, information acceleration) environment; it represents a spatial configuration

\footnotetext{
${ }^{1}$ Throughout this paper, we focus on an individual-level analysis of paths. Hence, macro-level modeling of paths, e.g., fluid dynamic models (Henderson 1974) or cellular automata descriptions (Chopard et al. 1996) are not considered.
} 
that contains all possible locations that can be realized. ${ }^{2}$ The existence of a physical/simulated environment is an important feature of path data that differentiates them from other sequence data (e.g., brand choice data), where an explicit spatial environment is not available. Note that in this paper, we focus on only spatial configurations that are observable. Thus, the "paths" that occur in latent space, e.g., a consumer's change of lifecycle state in a hidden Markov model (e.g., Du and Kamakura 2006), are not considered. $S$ may be continuous, i.e., a fixed subset of $\mathfrak{R}^{r}$ that contains an r-dimensional rectangle of positive volume (Banerjee et al. 2004), or it can be discrete, in which case it would be defined by a graph ${ }^{3}$. We discuss this differentiation in detail in Section 2.1.2.

The second component of the three-tuple, $A$, denotes a conscious agent who is making the movements. Since we focus on the movement made by a conscious agent, other path-related research areas such as robot paths (e.g., Thrun et al. 2005) or hurricane paths (e.g., Bril 1995) are not considered in this paper. While both of the above areas contain many useful insights, we believe that our current focus on conscious agents is reasonable given that our eventual goal is to analyze paths made by consumers in various marketing contexts.

Once $S$ and $A$ are specified, $X_{A}$ denotes the movement of the agent. Specifically, $X_{A}(t)$ represents the position of the agent $A$ at time $t$ within the spatial configuration. For $P$ to be considered a path, $X_{A}(t)$ must vary "continuously" over time with respect to the spatial configuration. If $S$ is continuous, the continuity of $X_{A}(t)$ can be defined as the conventional definition of continuity in mathematical analysis (Rudin 1976), given by:

\footnotetext{
${ }^{2}$ Note that our definition of path is on the underlying process, not on its measurement. The particular measurement of a path is subject to measurement error and limits in the sampling frequency.

${ }^{3}$ A graph is a mathematic object which consists of "nodes" and "edges". Nodes correspond roughly to different locations; an existence of an edge between two nodes indicates that it is possible to move from one node to the other in one step. For details, refer to Bollobas (1979).
} 


$$
\lim _{\Delta t \rightarrow 0} X_{A}(t+\Delta t)=X_{A}(t)
$$

On the other hand, if $S$ is discrete (a graph), $X_{A}(t)$ must vary in a way that is consistent with the structure of the graph; i.e., subsequent moves must be between nodes connected by an edge. ${ }^{4}$ This important definitional element marks the key difference between path data and other kinds of space-time data. More specifically, spatio-temporal datasets such as the product diffusion data in Bell and Song (2007) do not belong to path data under our definition. In product diffusion data, the data are not continuous: two successive adopters can come from very distant locations. ${ }^{5}$ Thus, although those data also have both spatial and temporal elements, they do not conform to the aforementioned continuity restriction and are thus not considered to be path data.

In the following discussion, we outline the two key components of a path model: the spatial configuration $S$ and the role of the agent $A$, and discuss the specific sub-dimensions upon which data considerations (and thus model specifications) may differ.

\subsection{Spatial configuration $(S)$}

As we discussed, the spatial configuration defines the space in which movements are made and specifies the set of allowable movements given an agent's current location. We characterize a spatial configuration along three separate sub-dimensions: (i) physical/nonphysical, (ii) continuous/discrete, and (iii) the presence/degree of constraints. Figure 2 illustrates spatial configurations in six different settings.

\section{[Insert Figure 2 about here]}

\subsubsection{Physical/non-physical}

\footnotetext{
${ }^{4}$ In the graph theory literature, this defines a "walk". We call it a "path" in our notation since it is more consistent with the terminology used in our exposition and in the marketing literature.

${ }^{5}$ One could argue that if there is a fixed and known social structure that connects different consumers, then the continuity restriction may be met. In that case, however, the data correspond to the movement of multiple agents, instead of an individual agent which is the focus of this paper.
} 
A spatial configuration may or may not correspond to an actual physical space. As examples of physical spaces, in a model of bird movements, the space is the three-dimensional sky; for eye-tracking data, it is the two-dimensional physical image that the subject is viewing. Pedestrian movements also employ a space that is generally limited to two dimensions; likewise for a grocery shopper, although the space of movements also includes a number of fixed impediments (aisles, display cases, etc.), which we will discuss shortly.

Some path data in marketing, however, do not take place in a physical environment, but instead in a computer-simulated environment (Kalawsky 1993; Hoffman and Novak 1996); examples include clickstream data and path data from information acceleration sessions. In these cases, we often need to generalize the notion of "space" to incorporate non-physical spatial configurations. This requires a careful mathematical depiction. For instance, Montgomery et al. (2004) built a discrete spatial configuration for web browsing: a graph consisting of eight nodes, with each node representing one of the eight online web "categories", as shown in the top right panel of Figure 2. Using this representation, each category can be viewed as a location on a (hypothetical) map defined by webpages, and therefore each consumer's clickstream sequence can be treated as a path on this map. This convenient representation is frequently used to model the structure of the World Wide Web (Broder et al. 2000; Eirinaki et al. 2005). Similarly, we can construct a graph, as shown on the bottom left panel of Figure 2, to represent the spatial configuration of data collected from an information acceleration session. Each location on the graph represents a source of information available to the consumer. Thus, the consumer's sequence of information viewing can thus be seen as a path on this graph. To the best of our knowledge, this characterization has never been used to analyze and describe information acceleration data. 


\subsubsection{Continuous/discrete}

As mentioned earlier, a spatial configuration can either be continuous or discrete. In models of birds and pedestrians, the spatial configurations are naturally continuous. In contrast, the spatial configurations for clickstream data or information acceleration are inherently discrete, since, as above, the space is constructed as a finite-node graph.

In some applications, however, the modeler can decide whether a continuous or a discrete representation is more suitable. For example, the space for eye-tracking data can be modeled as continuous if we use the entire advertisement as the space, and model the movement of the visual focus between two pairs of $(x, y)$ coordinates (see Figure 3, upper left panel). Alternatively, it can be modeled as discrete if we characterize the space as the different elements (e.g. picture, brand logo, text) in the advertisement, and model the transition of a consumer's visual focus among them (Pieters \& Wedel 2004), as depicted in the lower left panel of Figure 3. Similarly, we can define the space of grocery shopping as continuous by defining the space in terms of $(x, y)$ coordinates in the store, or we can instead define it as discrete by modeling shopper's movement between zones or departments (see upper and lower right panel, Figure 3). This “discretization,” approximating a continuous object by a mesh of discrete points, is similar to the use of the "finite element method” in engineering (Zienkiewicz et al. 2005) and is related to aggregation issues or (“wombling”) in spatial statistics (Banerjee et al. 2004). On the one hand, discretization may reduce the complexity of the data and thus facilitate computation; on the other hand, it is often not clear what level of aggregation is most suitable for the problem at hand. We return to this issue and discuss the relative advantages of a discrete versus continuous spatial specification in more detail later, when we outline model-building issues for marketing researchers.

[Insert Figure 3 about here] 


\subsubsection{Presence/degree of constraints}

Constraints may be present in a spatial configuration, which restrict the set of allowable movements that an agent, $A$, may take. In a physical setting, constraints are usually characterized by physical impediments. For example, aisles and walls in a grocery store restrict the possible direction of movement for grocery shoppers. Constraints may be less severe in other settings. For instance, in a model of pedestrian movement, the only constraints that a pedestrian faces is that he/she cannot walk into physical objects; other than that, an allowable movement is any location within the circle with the person's current location as the center, and a radius equal to $v_{\max } \Delta t$, where $v_{\max }$ is the person's maximum walking speed (Helbing et al. 1997). Likewise, physical constraints are generally absent in eye-tracking studies.

Note that in physical spatial configurations, there is a natural relationship between the degree of constraints and whether the space should be treated as continuous or discrete. In general, it is more appropriate to model space as discrete when movements are highly constrained. That is, movements are viewed as a sequence of choices among a finite set of alternatives, rather than a choice among infinitely many directions. As an example, in the pedestrian model, due to the lack of constraints, it may be more appropriate to model movements as occurring continuously in space; in contrast, for grocery shopping, it may be more appropriate to model the paths as transitions between different store “zones” (Hui et al. 2007).

Constraints may also exist in non-physical settings. If we represent a non-physical setting as a graph, the presence of constraints between two points implies the absence of edges between the respective nodes that represent them. For instance, in Montgomery et al. (2004), at each step the user is located at the node that represents his current category, and he is allowed to move to any node that is “connected”. As can be seen on the top right panel of Figure 2, the 
"product" node and the "order" node are not directly connected. This implies an existence of a constraint — a person cannot directly reach the "confirm order" node from the product node in one step, since he has to go through the "shopping cart" node in between. In information acceleration sessions, constraints are largely absent and the agent is relatively free to move to any location; albeit, there are sessions set up in such a way that not all possible movements are available in one step.

\subsection{The agent (A)}

Having defined a spatial configuration, researchers need to characterize the conscious agent who is making the movements. For most path models, the definition of an agent is usually straightforward. For example, the agent for a grocery shopping trip is the shopper; for a clickstream model, the agent is the user browsing the internet. Sometimes the agent is a welldefined group, e.g., a family traveling together in a car. Complications can arise if a multi-unit agent "takes turns," e.g., a parent who lets a child push the grocery cart, or if the data collection process loses track of specific entities (which can conceivably occur with birds, for instance). One of the clear advantages of experimental settings (such as information acceleration and eyetracking) is the high degree of control that the researcher maintains in this regard.

Beyond these definitional aspects, there are three key sub-dimensions that should be considered for each agent: (i) social interaction, (ii) goal-directedness, and (iii) forward-looking tendencies, all active research topics in marketing today.

\subsubsection{Social interaction}

When the agent co-occupies the same spatial configuration with other agents, social effects may be present. That is, an agent may be affected by the other agents' actions when deciding his next movement. For instance, although each bird in a flock is autonomous, it tends 
to match the velocity of its nearby flockmates to avoid collision, while staying close to the center of the flock (Reynolds 1987). Similarly, researchers found that pedestrians have strong tendencies to preserve interpersonal spaces to avoid running into each other (Helbing et al. 1997). The existence of social interaction between shoppers is especially relevant in the physical retail environment. For instance, researchers found that grocery shoppers typically avoid walking into crowded areas (Harrell et al. 1980), and have demonstrated that even the "mere social presence” of others will affect shopping behavior (Argo et al. 2005). In particular, consumers may reduce their shopping time (Harrell et al. 1980) or even refrain from purchasing a product if they feel that their personal space is "invaded” (Underhill 2000). On the other hand, consumers may exhibit a certain "herding" behavior by moving towards areas where other shoppers are heading, presumably because shoppers may infer the attractiveness of a store location based on other shopper’s actions (e.g., Banerjee 1992). For instance, Becker (1991) documented that, when given the choice between two almost identical restaurants, people usually prefer to choose the one with a longer line despite the longer wait. This leads to an interesting dynamic: while retailers should carefully design their store to avoid overcrowding conditions, they may also want to make sure that certain areas do not receive "too little" traffic. Thus, social interactions between shoppers may be an important issue to be considered when designing a retail environment.

In some cases, however, social effects may be insignificant. In particular, web browsing, participation in an information acceleration session, and reading an advertisement during an eyetracking study are all individual activities that, by design, involve little interactions among agents. Thus, Montgomery et al. (2004) did not choose to consider social effects in their model of clickstream data. Similarly, researchers can ignore any social effects when modeling path data 
from eye-tracking and information acceleration sessions; albeit, one may wonder whether movements in both of these cases would be influenced by social norms (of others), and/or expectations by the participant as to what he/she is "supposed" to look at (social cues).

Information acceleration commonly attempts to bring in social cues through personal testimonials, which can influence the subsequent paths chosen by the subject. This is an intriguing aspect of technology, particularly when these testimonials (and the characteristics of the person(s) delivering them) are manipulated experimentally. But while the cues may affect subjects' movement, there is no provision for direct social influence in current applications of information acceleration. This may be possible, however, by including statements such as " $80 \%$ of previous users clicked on area Y after visiting this page.” Participants may become more inclined to view area $\mathrm{Y}$ after viewing this suggestive statement.

\subsubsection{Goal-directedness}

An agent may be goal-directed (Lee and Ariely 2006) and begin the path process with specific goal(s) in mind (e.g., to buy groceries for dinner, to find a textbook on Amazon.com), or at the other extreme, his trip can be a purely hedonic "browsing” experience. Agents with varying degrees of "goal-directedness" may exhibit very different paths. For instance, in her study of online shoppers, Moe (2003) identified four types of internet browsers with different goals, “directed buying,” "search and deliberation,” "knowledge building,” and "hedonic browsing," and found that distinct types of shoppers exhibit very different page-to-page browsing patterns (paths). This distinction can be extended to other areas that involve paths. For example, a grocery shopper armed with a shopping list probably follows a movement pattern that is different from another shopper who plans to do some "window shopping." Likewise, a person reading a magazine to find product information may exhibit an eye-movement pattern that is 
distinct from that resulting from leisurely reading a magazine article. In online settings, web users also exhibit different degrees of goal-directed behavior. For instance, Montgomery et al. (2004) allowed each subject to be in either a "browsing" or a "deliberative" latent state; in each state, the subject exhibits different search behavior. Thus, to accurately specify a model of movements, researchers must carefully define what goal-directedness means in each context and capture differences across agents on this dimension. This is an example of heterogeneity among agents, an issue we will return to in Section 4.

Note, however, that path data alone does not always give us reliable indications of the mood, goals, and intentions of the agents; thus, whenever possible, we recommend that other methods (e.g., surveys) be used in conjunction to path data to capture that information.

\subsubsection{Forward-looking behavior}

A goal-directed agent can be forward-looking (e.g., Song and Chintagunta 2003; Sun et al. 2003); that is, along his path, an agent may plan ahead for his subsequent actions. Researchers have considered various degrees of planning-ahead behavior in many path settings. For instance, Hui et al. (2007) studied how a grocery shopper plans ahead the order in which he gathers products on his shopping list. At a more macro level, researchers in the "trip chaining" literature studied how consumers plan ahead to "chain” together different errands which take place in different locations (e.g., Adler and Ben-Akiva 1979).

Forward-looking behaviors are manifested not only in retail settings but also in situations that involve information search. A web user may have a specific plan of search/query in mind when trying to satisfy his information needs (Robertson 1977). For example, a person whose goal is to purchase a digital camera may first plan on collecting information on the types of cameras available, then specific features (e.g., resolution, battery life), then decide on the exact 
model and finally conduct a price search on that model. Even in a non-purchase setting such as information acceleration, a person may plan ahead on how he wants to evaluate the products/services of interest; for instance, he may plan to first pay attention to the objective features of the products, before taking into account the more subjective information such as product reviews and word-of-mouth information.

While the extent of plan-ahead behavior is an important characteristic of the agent, different agents may exhibit different degrees of forward-looking tendencies. Thus, to realistically capture consumer behavior, path modelers need to specify and incorporate different degrees of forward-looking behavior. To that end, we believe that researchers can benefit by exploiting the close connection of plan-ahead behavior to many classical network optimization problems in operations research, as we outline briefly in the following example taken from Hui et al. (2007).

Consider a grocery shopper who carries a shopping list. Assuming that he wants to take the shortest path that allows him to gather his purchases, we can view that the shopper is solving a “Traveling Salesman Problem” (TSP) (Lawler et al. 1985). Since the traveling salesman problem is a very complex combinatorics problem, this specification clearly assumes too much knowledge and computational ability on the part of the consumer. As a result, a model that assumes consumers follow the path suggested by the optimal solution of the corresponding TSP problem will provide a poor fit to the actual data.

To develop a more realistic descriptive model, researchers may consider two different relaxations that correspond to two different extant research areas in operations research. First, if we assumes that a shopper obtains information about the product placements as he moves around, the problem will be isomorphic to a variant of the TSP known as the “online TSP” (e.g., Ausiello 
et al. 2001), where information about the environment is released based on a certain "schedule".

Second, if we reduce the computational capabilities on the part of the consumer, we may capture the consumer's planning behavior by a greedy algorithm (i.e., he moves towards the location which carries the item on his shopping list that is not yet purchased and is closest to his current location), or more generally by a greedy algorithm with a certain number of look-ahead steps (Cormen et al. 2001). These extensions allow researchers to borrow methods from the operations research literature to build models of paths.

\section{Literature Review}

Based on the framework developed in Section 2, we provide a literature review for path data that arise in four different areas in marketing: retail/service environments (Section 3.1), advertising studies (Section 3.2), e-commerce (Section 3.3), and experimental research (Section 3.4). We also briefly outline some path-related areas that are outside of marketing in Section 3.5.

\subsection{Paths in retail/service environments}

Marketing researchers have tracked consumers’ movements in various retail settings, including grocery stores (Heller 1988), shopping malls (Underhill 2004), and museums (e.g., www.crowddynamics.com). The earliest study in this area is Farley and Ring (1966), who physically followed grocery shoppers and documented their movement patterns. Since then, advances in data collection technology have provided researchers with an increasingly sophisticated set of tools to track consumers' movements. Hidden cameras and motion sensors are used to monitor traffic flow (Gogoi 2005), and even capture consumers' eye movements and facial expressions (Pereira 2005). Researchers have also built computer-simulated grocery stores to study people’s shopping patterns (Burke 1996; Vrechopoulos et al. 2004). More recently, Radio Frequency Identification (RFID) technology has been used to monitor in-store traffic (Hui 
et al. 2007; Sorensen 2003). Other promising tracking technologies include the use of Global Positioning Systems (GPS) and portable shopping devices. Interested readers are encouraged to see Burke (2005) for a comprehensive overview of these new technologies.

To date, researchers have performed various exploratory analyses on shopping paths. Heller (1988) measured the flow of shoppers to each department of a grocery store, and tabulated the pattern of browsing and purchasing at each product aisle. Underhill (2004) qualitatively documented how shoppers interacted with the store environment, then made recommendations to improve shopping convenience. Otnes and McGrath (2001) tracked the shopping paths of male shoppers to test hypotheses about gender-specific shopping behavior. Based on RFID tracking technology, Sorensen (2003) developed a shopper-tracking system known as PathTracker ${ }^{\circledR}$; his firm has collected and analyzed over 200,000 shopping paths. Larson et al. (2005) later performed a K-medoids clustering algorithm on a sample of these path data and identified a total of 14 different patterns (clusters).

The above research on shopping paths have focused on analyzing paths that occur within a certain environment, such as a grocery store or a shopping mall. In contrast, researchers in the area of "trip chaining" (e.g., Thill and Thomas 1987) studied the paths that people take when they perform a set of errands that take place in different geographical locations. Researchers studied how people select the order of visiting those different destinations, and how they choose the mode of transportation in each trip segment. (Adler and Ben-Akiva 1979). For example, Dellaert et al. (1998) investigated how consumers sequence shopping trips that involve three separate destinations (grocery, drugstore, and clothing store). See Thill and Thomas (1987) for an overview of research on trip-chaining behavior.

3.2 Paths in advertising studies 
In advertising studies, a technology known as infrared (or near-infrared) corneal reflection is often used to trace a subject's eye movements (Duchovski 2002). During the study, an infrared beam, invisible to the eye, is reflected off the cornea, and its angle of reflection is continuously monitored. The visual focus of the eye at any moment can then be calculated thus the technique is often referred to as "eye tracking."

In psychology, eye tracking has been widely used to study people’s reading and information processing patterns (Rayner 1998). In marketing, researchers have applied eye tracking methodologies to capture consumers' eye movements when they view print advertisements (e.g., Janiszewski 1993; Krugman et al. 1994; Pieters et al. 1996, 1999, 2002; Rayner et al. 2001; Rosbergen et al. 1997). For instance, Pieters and Wedel (2004) followed consumers' visual focus and used that as a proxy for the amount of attention paid to the different elements (brand, pictorial, and text) of an advertisement. They then studied how different sizes of each element enhance or reduce overall visual attention. In the same spirit, Fox et al. (1998) studied the effectiveness of different alcohol and cigarette warning messages by tracking the amount of time consumers' focus (visually) on the messages and offered practical managerial insights to improve the effectiveness of health warnings.

Marketing researchers have also tracked consumers' visual movements when they are reading yellow page advertising (Lohse 1997), searching the web for information (Goldberg et al. 2002), and while making brand choice decisions (Chandon et al. 2001; Pieters and Warlop 1999). Other researchers have utilized eye-tracking techniques to study the effect of flash banners (Day et al. 2006), to assess the influence of page layout on visual search pattern (Janiszewski 1998), and even to infer the acceptability of brand extensions (Stewart et al. 2004).

\subsection{Paths in e-commerce}


In e-commerce, marketing researchers collect “clickstream” data, which contain detailed click-to-click page-viewing information for each consumer (Bucklin et al. 2002). Montgomery et al. (2004) used clickstream data, together with a dynamic multinomial probit model that captures page-to-page transitions, to predict purchase conversion. They reported that their model performed better than traditional models that failed to take full advantage of the richness of the path data. Likewise, Moe et al. (2002) and Sismeiro and Bucklin (2004) predicted consumers' online purchasing behavior more accurately by using browsing characteristics as covariates.

The potential applications of the analysis of clickstream data extend beyond the prediction of purchase behavior; researchers also study web surfing patterns to understand users' information search behavior and classify users' web browsing strategies. For instance, Chi et al. (2001) developed the idea of “information scent” to infer a user’s information needs given his pattern of surfing. Moe (2003) defined and tabulated 14 different summary statistics of each page-to-page viewing session, and used them to classify a visit as a buying, browsing, searching, or information-building one. Similarly, Catledge and Pitkow (1995) recorded user behavior to understand navigational strategies and made suggestions on web design to enhance usability. Other marketing applications and research opportunities are outlined in Bucklin et al. (2002). Several advances in the analysis of clickstream data have also been made in computer science, which can potentially be applied in marketing in the future. For instance, researchers developed new data-mining methods to uncover common path traversal patterns in order to predict a browser's next choice of webpage given his viewing history (Li et al. 2004). Other researchers clustered web users based on their shared interests inferred from their sequence of webpage visits and hyperlink selections (Shahabi et al. 1997), and subsequently profiled consumers based on their navigation patterns (e.g., Banerjee and Ghosh 2000, 2001). Computer 
scientists have also learned patterns from clickstream data to customize webpage content (Mobasher et al. 2001) and to generate product recommendations using collaborative filtering (e.g., Kim et al. 2004). These and several other applications are discussed in Theusinger and Huber (2000).

\subsection{Paths in experimental research}

Path data also arise naturally in experimental settings such as information acceleration (Urban et al. 1997) and Mouselab (Payne et al. 1988). Information acceleration (IA) belongs to a broad class of multimedia-based testing techniques (Hoffman and Novak 1996) and is often used to predict the success or failure of a new product before it is fully developed. During an IA session, consumers are first "accelerated" into a future context that conditions them to understand the environment surrounding the new product being tested (e.g., a world with a severe gas shortage). They are then instructed to gather information to decide whether/when to purchase the new product (e.g., an electric automobile). They learn about the new product by viewing, in any order and frequency they choose, a set of simulated product information, such as product attributes, product photos, virtual showroom visits, videos, advertising, newspaper articles, and (simulated) consumer reviews. The path of information viewing and time durations are recorded by the researcher to study consumers' information acquisition and processing patterns (Hauser et al. 1993). Very often, an experimental design manipulates the kinds of information seen by different consumers (e.g., some see positive word-of-mouth testimonials, while others see negative ones) and researchers can observe how this impacts consumers’ subsequent information collection (and product purchase) decisions.

Another example that involves path data includes Mouselab (e.g., Payne et al. 1988; Sen and Johnson 1997), a mouse-based computer system used by psychologists and marketers to 
study the process of how a person acquires information and makes decisions. With the Mouselab software, researchers are able to trace a subject's information acquisition movement by recording the movement of the mouse as a subject answers a series of questions. The resulting data reflect the path of the mouse pointer over time. Other related examples include the use of virtual reality systems that allow users to interactively explore simulated environments, usually to learn about a product (e.g., Volkswagen cars, Sprint telephones) (Lurie and Mason 2007).

Although a system such as Mouselab can generate very interesting path data, we have never seen it utilized in such a manner. Past researchers have tended to rely on more traditional analyses, e.g., two-sample t-tests and aggregate summary statistics. Well-specified models built upon the entire Mouselab path data may offer additional insights about the observed behavior and the cognitive processes underlying them.

\subsection{Paths outside of marketing}

Outside of marketing, many researchers have also collected and analyzed path data. Researchers have studied the movement of animals (e.g., Polovina et al. 2000; Preisler et al. 2004), insects (e.g., Crist et al. 1992; Jeanson et al. 2003), and pedestrians (e.g., Helbing and Molnar 1995; Teknomo et al. 2000). Reynolds (1987) modeled the motion of a flock of birds using simple behavioral assumptions. He assumed that each bird moves according to three simple rules: (i) it attempts to move closer to the other birds in its neighborhood; (ii) it matches the velocity of its flockmates; (iii) it avoids collision with other birds. Using computer simulations, Reynolds (1987) was able to replicate realistic flock motions.

In a similar vein, Helbing and Molnar (1995) proposed a model based on an analogy between people's movement and the principles of Newtonian mechanics. They defined and modeled latent "social forces" that can be exerted on an individual. In their model, social forces 
that act on a person may include the "attractive" force exerted by one’s destination, "repellent" force from other pedestrians to avoid collision, and the attractive forces caused by the inherent "comfort" of the ground. The aggregation of these forces in turn governs the direction of a pedestrian's movement.

While some of these concepts may not apply directly to many consumer settings, they give us thought-provoking metaphors that help us derive our framework and offer new ways to think about patterns of shopping, information search, and e-commerce.

\section{Issues in Building Models of Path Behavior}

In this section, we discuss some modeling issues/choices that researchers will frequently face when developing path models. We outline the issues in parallel with the structure of our framework, by first discussing modeling issues for the spatial configuration, the agent, and finally, the paths.

4.1 Modeling the spatial configuration

Modeling a spatial configuration as a discretized grid, as compared to a continuous space, can lead to several advantages. First, discretization simplifies the space which may originally contain an infinite number of locations and thus reduces computational complexity (see the two examples in Figure 3). When the sample size is large (e.g., Sorensen 2003 collected 200,000 grocery shopping paths; Larson et al. 2005 analyzed a sample of 27,000 paths), this reduction in complexity may be crucial to enable implementation. Second, since a consumer faces a finite number of alternatives at each step, a discrete spatial configuration ties well with discrete choice models (e.g., Guadagni and Little 1983) used widely in marketing. In contrast, with a continuous space, researchers will need to develop/utilize a different class of choice models that can handle an infinite number of alternatives. Third, if the discretization is made carefully based on 
substantive insights, such models will allow for easier interpretation. For instance, if a grocery store is discretized based on the locations of product categories, managers can readily interpret and understand the output by relating them to category-to-category transitions; on the other hand, a continuous model will probably not be as directly linked to substantive insights or managerial actions.

While discretization leads to many advantages regarding computation, model building, and interpretation, it does have some limitations. First, the process of discretization requires one to re-code the original continuous $(x, y)$ data into discretized form. This data preparation step may be very time consuming if the dataset is large or the spatial environment is complex. Second, discretization requires careful construction of a graph, based on substantive insights, to represent the underlying spatial configuration. In problems where little domain knowledge exists, researchers may find it difficult to construct a reasonable representation. A related concern is that the conclusions derived from the path model may vary based on the chosen method of discretization. Thus, we recommend that researchers experiment with different discretization schemes to see how sensitive model conclusions are to different specifications. Finally, the process of discretization reduces the resolution of the data. Since we are approximating continuous data using a discrete grid, variations in the original data at a level finer than the grid will be lost. Thus, researchers may also want to experiment with different levels of granularity in the discretization to determine the “optimal” resolution.

\subsection{Modeling the agent}

To model the characteristics of the agent, two particularly important research issues need to be considered. First, researchers need to specify the role and the nature of the heterogeneity 
among agents. Second, since agents’ behavior may change over the course of their path, adaptive/non-stationary behaviors should also be taken into account.

\subsubsection{Role(s) of heterogeneity}

Researchers need to specify the dimension(s) on which consumers are considered to be heterogeneous in order to calibrate their path models. This issue requires careful thought because consumers can be heterogeneous in many different aspects, including all of the dimensions we have discussed, i.e., social interactions, goals (if any), and forward-looking tendencies.

For instance, consumers may exhibit different responses to social interaction; researchers have documented that even given the same in-store shopper density, people may have different perceptions of how "crowded” the area is. Perceived crowding is influenced by both individual characteristics and situational factors (Harrell and Hutt 1976). In addition, consumers may respond differently to crowding situations: while some may shorten the time they spend choosing between products, others may become more goal-directed and spend less effort in exploration.

Likewise, consumers may enter the spatial configuration with different goals. In a grocery store, for example, each consumer may come into the store with a different set of product categories that he wants to purchase; some shoppers, who may be hedonic browsers or window shoppers, may not have a clear set of purchase goals. Researchers may consider capturing goal and social-interaction heterogeneity by specifying a set of individual-level parameters that describe such behavior and linking them via a Hierarchical Bayes framework (Rossi et al. 2006), or by segmenting consumers into different classes, based on their social behavior and goal-directedness, using a latent class model (Kamakura and Russell 1989).

Accounting for heterogeneity in consumers' forward-looking behavior requires even greater thought because of the difficulty in representing forward-looking behavior as a 
"parameter." As we discussed in Section 2.2.3, researchers may exploit the close connection between planning behavior and many classical network optimization problems (Cormen et al. 2001). This connection may allow researchers to experiment with a wide array of model specifications based on different assumptions of consumers' knowledge of the environment (e.g., Moorman et al. 2004), their degree of rationality, and the optimization algorithm that they are presumed to follow. Researchers can then test these model specifications with field data from a panel of consumers with different levels of knowledge about the spatial environment. For instance, based on an analogy with the Traveling Salesman Problem, Hui et al. (2007) analyzed the efficiencies of grocery shoppers to infer their number of look-ahead steps, similar to Camerer and Ho (1999) in their study of behavior in experimental economics games.

\subsubsection{Adaptive/nonstationarity behavior}

Researchers also need to take into account the fact that a path process may not necessarily be stationary. That is, as consumers progress in a trip, they may exhibit some degree of adaptive/nonstationary behavior. First, some changes may occur naturally as part of the path process. For example, a web user may naturally switch from an initial browsing state, when he is casually surfing the web for general information, to a deliberation state, when he decides that he wants to purchase certain products (Montgomery et al. 2004). Similarly, Bates (1989) hypothesized a new model of information search called "berrypicking,” which assumed that users obtain information “a bit at a time”, and their information need evolves over time based on the previous search results. In both cases, the consumers' goals have changed over time and must be carefully modeled.

Second, some changes may accumulate gradually over the path. For instance, a grocery shopper may become more fatigued the longer she spends in the store. Thus, we may observe 
that as time progresses, the shopper may want to spend less time exploring the store and instead use a more directed approach to pick up only those products that she needs. Indeed, Sorensen (2003) documented that shoppers tend to speed up the more time they spent in the store. Finally, some changes may also be driven by social influence, as consumers adapt their behavior based on what other consumers do, e.g., to avoid crowding or to preserve interpersonal space (Underhill 2000).

These different types and sources of nonstationarity are important because they may necessitate different modeling methodologies. For changes that happens suddenly and involve a discontinuous change of “state,” approaches such as Hidden Markov models (Rabiner 1989), or more generally state space models (Kim and Nelson 1999), where consumer behavior is modeled conditional on their hidden states (which may evolve over time through some stochastic processes), may be more appropriate. On the other hand, if the changes are gradual, it may be more appropriate to incorporate the continuous change as a parameter in the model and allow for a "trend" component on that parameter to capture its smooth time-varying nature.

\subsection{Analysis and modeling of paths}

We now discuss the following issues that arise in the analysis and modeling of paths. First, we describe some statistical issues involved in exploratory analyses of paths. Second, we discuss the deterministic/stochastic nature of movements. Finally, we explore the challenges in building models that integrate data collected from paths and other behaviors (e.g., purchases).

\subsubsection{Statistical issues for exploratory analyses}

Before developing a formal model of paths, it is helpful for a researcher to graphically display the data and perform exploratory analyses (Wainer 2004). Performing exploratory analyses on paths, however, is non-trivial and can pose major challenges for marketing modelers 
and statisticians. By nature, path data are multivariate and therefore relatively complicated: each record in a path dataset is a multivariate sequence that represents the consumer's position over time. In addition, path data are also fundamentally different from traditional multivariate data because, as we discussed in our definition of paths, there is a continuous relationship between each position over time. Thus, commonly used multivariate techniques (e.g., cluster analysis, factor analysis) that ignore this temporal relationship may not be suitable. Instead, statisticians and modelers need to consider a number of key statistical issues, such as:

(i) How should we display and compare paths that are different in sampling intervals? For example, some paths may contain record movements in 5-second intervals, while others in 15-second intervals. How should we store and format this kind of data?

(ii) How should we compare paths that are different in duration? For instance, a shopper who spends two hours at a grocery store will have a much longer path than a shopper who spends five minutes. How do we create a metric/topology of paths that will allow us to calculate the "distance" between these paths?

(iii) How can we summarize the sources of variation among paths, taking into account constraints within the spatial configuration (e.g., walls, aisles)?

Larson et al. (2005) approached the last problem using a K-medoid clustering technique applied to grocery cart movement data. It would be interesting if, in addition to obtaining a clustering solution, we could describe path data using ideas from principal components analysis (PCA) to break each path into some combination of lower-level components. For instance, Bradlow (2002) described an approach to apply PCA to repeated measures data sets to explore their key features. With some modifications, researchers may be able to similarly extend PCA methods to extract key information from path data. 
More generally, we believe that functional data analysis (Ramsay and Silverman 2005) seems to provide another appropriate set of tools to address these issues. In functional data analysis, the underlying data are functions themselves; path data are functions of time, with the function value at each time point being the agent's "location.” Although many sophisticated exploratory techniques have already been developed in functional data analysis, they cannot be directly applied to path data. This is mainly because the existing methodology typically assumes that the underlying space is unrestricted, while path data often contain spatial constraints. Thus, identifying a proper method to handle these constraints is a crucial issue for those who wish to apply functional data analysis techniques to path data.

Another major challenge in exploring path data is how one should take into account other ancillary information available for each path. For example, a dataset of grocery shopping paths not only includes the shopping path information but also typically will be linked to purchases. Since the data comes in two different forms: a path (functional data) and a record of purchases (multivariate data), existing exploratory techniques (e.g. clustering) would be ill-suited to describe the relationship. One promising direction is through the use of a mixed-PCA algorithm developed in functional data analysis which may allow us to analyze datasets containing both functional and multivariate data (Ramsay and Silverman 2005).

\subsubsection{Deterministic vs. stochastic}

The law of motion for a given model, i.e., the exact mechanism with which movements are determined, given all the characteristics of the agents and the spatial configuration, can be deterministic or stochastic. In areas outside of marketing, researchers commonly use a deterministic specification to model the movement of birds and pedestrians. Given an initial configuration of birds and their migration target, the path of the flock is generally assumed to be 
completely determined (Reynolds 1987). In the social force model for pedestrians, a "potential surface” is calculated from the vector summation of all social forces acting on a pedestrian, which reflects the attractiveness of each location (Helbing et al. 1997). Given this potential surface, the pedestrian is assumed to move in the direction with the largest increase in ground attraction, much like how particles move in a force field.

In marketing, in contrast, a stochastic framework is often used to model movements. For instance, Montgomery et al. (2004)'s clickstream model specifies that the utility of a category is a linear function of covariates and a vector-autoregressive (VAR) structure, plus a (multivariate) normally distributed error term. These types of components are commonly employed in random utility models used in econometrics; it allows for the non-deterministic nature of click-to-click level web browsing patterns, even after accounting for all observed and unobserved covariates. Likewise, models of eye-tracking "scanpaths" often assume that the path of visual movement is stochastic, and is usually modeled using multivariate logit-type choice models (Pieters and Wedel 2004).

The fusion between deterministic and stochastic formulations leads to many intriguing opportunities for modeling. While a stochastic formulation is more flexible and consistent with random utility models in marketing, we believe that the introduction of some deterministic components within a general stochastic formulation may allow for a richer and more structural model of movement. For instance, researchers have documented that pedestrians tend to make right turns (Bitgood and Dukes 2006) and tend to speed up as they progress in a shopping trip (Sorensen 2003). To incorporate these and other general behavioral tendencies, we suggest that they can be modeled as deterministic components in a path model, while finer-level decisions can be modeled using stochastic terms. 


\subsubsection{Degree of integration for multivariate observed behaviors}

A random utility specification for a path model may facilitate the fusion between path models and other discrete choice models (e.g., purchase/brand choice). Such fusion has not been a hallmark of modeling work in this area and would be a significant contribution. In other domains, however, marketers have built integrated models of purchase incidence, brand choice, and purchase quantity (Chintagunta 1993), as well as the "who, when, where, and how much" characteristics of auctions (Park and Bradlow 2005). The integration of movement and choice data would be in the same spirit and offers many intriguing research opportunities.

One key issue to consider in this promising direction is the degree of integration for the observed behaviors. This issue is important because an integration of path and other behaviors only makes sense if they are strongly connected. This may hold in some settings; for example, one would assume that in a grocery store setting, a person will be more likely to visit the product aisle that he intends to buy from. Thus, the final market basket probably has a strong relationship with the path that the shopper takes. In other settings, however, the connection between one's path and the other behaviors may not be evident. For instance, a person who spends a long time looking for information about several books on Amazon.com may not actually buy from the website, but may be using it instead to gather information about a particular author. In a model of online conversion behavior, Moe and Fader (2004) found a considerable number of these "hard-core non-buyers" in the clickstream data from this website. In such a case, the connection between the path taken and purchases can be weak.

To study the degree of integration between paths and other activities, researchers may consider fitting two (nested) models: one that allows for the integration between the path and other activities (e.g., purchase), and the other that explicitly "turns off” this linkage by restricting 
some of the parameter values. The relative fit of the nested models allow researchers to understand whether a model that integrates different behaviors is superior. With more such exercises in the future, researchers will be able to make some empirical generalizations about the strength of the connection between different kinds of activities and the consumer's path to achieve them.

\section{Conclusion and Future Research Directions}

The main objective of this paper is to motivate and clarify research issues related to path data in marketing. Understanding the interaction between a consumer's behavior and his spatial environment allows us to extract vital insights about how and why consumers engage in certain behaviors. Using grocery shopping, web browsing, eye tracking, and information acceleration as primary examples, we find that marketing researchers have long been collecting and analyzing path data, but there has never been a general framework linking them. Thus, we outline a number of different areas where experimental and empirical researchers may collect and analyze path data, and synthesize these different areas by highlighting the role of paths in a unifying framework to guide future work in this broad area.

We offered an integrative framework of path models that pinpoints its various components and dimensions. We identified the two key components of a path model: the spatial configuration and the agent. The spatial configuration can be classified along three subdimensions: (i) physical/non-physical, (ii) continuous/discrete, and (iii) the degree of constraints. Agents can be characterized based on (i) their degree of social interaction, (ii) their degree of goal-directedness, and (iii) their forward-looking tendencies. We then moved on to discuss an array of statistical and modeling issues that researchers might face in constructing a path model. 
While we do not claim to provide a complete list of challenges and issues, we hope to offer some useful guidance to future researchers faced with path-related datasets.

As path datasets become more commonly available, we expect to see more research activities in this new and promising area. These activities will not only include descriptive models of movements and choices (as highlighted in this paper), but we also expect to see a richer variety of research issues addressed. We briefly describe three possible areas that reflect the kinds of investigations we hope to see in the near future:

(1) Hierarchies of paths: In this paper, we have focused on agents operating in a single spatial environment. More generally, one may consider a "hierarchy" of paths. At a high level, a consumer decides the sequence of websites to visit (e.g., Park and Fader 2004). Within each website, he may then navigate among the webpages with a specific click-to-click pattern (e.g., Montgomery et al. 2004). Similar issues arise in a shopping mall or even a large store (Arentze et al. 2005; Brooks et al. 2004), where there may be a hierarchy of paths in effect (i.e., deciding which stores/departments to visit, then beginning a new path within each one). One can imagine how observations about the paths within limited parts of the hierarchy would be informative about the shopper's behavior in other parts of it.

(2) Inferences about partially-observed paths: As we learn more about path patterns, we might get to the point where we do not need to actually see all the movement data in order to make accurate statements about path-related behaviors. If we can tell a valid, empirically supportable "story" about the path, then we might need nothing more than a series of still images from key locations in the environment to capture the relevant aspects of the path (instead of relying on complete point-to-point movement records). The cost-savings would be very significant, and if the story is a decent one, then there may be limited loss of 
information in "throwing away" much of the movement data. Research firms such as Sorensen Associates are actively investigating such methods, and modeling techniques such as those developed by Musalem et. al (2006a, 2006b) make this a promising area of academic research.

(3) Convergence of different types of paths: Although we have carefully compared and contrasted various types of marketing paths throughout the paper, we have treated each one as a separate entity, without considering possible links among them. But as data collection technologies become more sophisticated and economical, we envision that there could be a convergence of different path domains; i.e., researchers may simultaneously collect and combine different data sources. Several current sources of convergence include: (1) companies that not only track typical page-to-page clickstream data, but also the "movie" of mouse movement within each page to see how users interact with a website (www.clicktale.com); (2) Touch screen kiosks in retail stores (or even on the store window - see http://wcbstv.com/technology/local_story_219114456.html) which combine elements of clickstream and in-store path data; and (3) Collecting eye-tracking data at the store shelf, which can be combined with customer movement data. All of these data sources (and countless other areas where convergence of path data might occur) will create more exciting challenges and opportunities for marketing researchers who wish to learn even more about different aspects of consumer behavior.

We encourage other researchers to join us in the study of paths to investigate these and other interesting issues. 


\section{References}

Adler, Thomas and Moshe Ben-Akiva (1979), “A Theoretical and Empirical Model of Trip Chaining Behavior,” Transportation Research, 13B, 243-257.

Arentze, Theo A., Harmen Oppewal, and Harry Timmermans (2005), “A Multipurpose Shopping Trip Model to assess retail agglomeration effects,” Journal of Marketing Research, 42(1), 109-115.

Argo, Jennifer J., Darren W. Dahl, and Rajesh V. Manchanda (2005), "The Influence of a Mere Social Presence in a Retail Context,” Journal of Consumer Research, 32, 207-212.

Ausiello, Giorgio, Esteban Feuerstein, Stefano Leonardi, Leen Stougie, and Maurizio Talamo (2001), "Algorithms for the on-line traveling salesman,” Algorithmica, 29(4), 560-581.

Banerjee, Abhijit V. (1992), “A simple model of herd behavior,” The Quarterly Journal of Economics, 797-817.

Banerjee, Arindam and Joydeep Ghosh (2000), "Concept-Based Clustering of Clickstream Data," Proceeding of the $3^{\text {rd }}$ conference on Information Technology.

Banerjee, Arindam and Joydeep Ghosh (2001), "Clickstream Clustering using Weighted Longest Common Subsequences," Proceedings of the Workshop on Web Mining, SIAM Conference on Data Mining, Chicago, IL, 33-40.

Banerjee, Sudipto, Bradley P. Carlin, and Alan E. Gelfand (2004), Hierarchical Modeling and Analysis for Spatial Data, Chapman \& Hall.

Bates, Marcia J. (1989), “The Design of Browsing and Berrypicking Techniques for the Online Search Interface," Online Review, 13(5), 407-424.

Becker, Gary S. (1991), “A Note on Restaurant Pricing and Other Examples of Social Influence on Price," Journal of Political Economy, 99, 1109-1116.

Bell, David, and Sangyoung Song (2007), "Neighborhood Effects and Trial on the Internet: Evidence from Online Grocery Retailing,” Quantitative Marketing and Economics, forthcoming.

Bitgood, Stephen and Stephany Dukes (2006), "Not Another Step! Economy of Movement and Pedestrian Choice Point Behavior in Shopping Malls,” Environment and Behavior, 38(3), 394-405.

Blackmore, Susan (2003), Consciousness: An Introduction, Oxford University Press.

Bollobas, Bela (1979), Graph Theory: An Introductory Course. New York: Springer-Verlag. 
Bradlow, Eric T. (2002), "Exploring Repeated Measure Data sets for Key Features Using Principal Components Analysis,” International Journal of Research in Marketing, 19, 167-179.

Bradlow, Eric T., Bart Bronnenberg, Gary J. Russell, Neeraj Arora, David R. Bell, Sri Devi Duvvuri, Frankel Ter Hofstede, Catarina Sismeiro, Raphael Thomadsen, and Sha Yang (2005), “Spatial Models in Marketing,” Marketing Letters, 16, 267-678.

Bril, G. (1995), “Forecasting Hurricane Tracks Using the Kalman Filter,” Environmetrics, 6(1), 7-16.

Broder, Andrei, Ravi Kumar, Farzin Maghoul, Prabhakar Raghavan, Sridhar Rajagopalan, Raymie Stata, Andrea Tomkins, and Janet Wiener (2000), "Graph Structure of the Web," Computer Networks, 33, 309-320.

Brooks, Charles M., Patrick J. Kaufmann, and Donald R. Lichtenstein (2004), "Travel Configuration on Consumer Trip-Chained Store Choice,” Journal of Consumer Research, 31, 241-248.

Bucklin, Randolph E., James M. Lattin, Asim Ansari, Sunil Gupta, David Bell, Eloise Coupey, John D.C. Little, Carl Mela, Alan Montgomery, Joel Steckel (2002), "Choice and the Internet: From Clickstream to Research Stream,” Marketing Letters, 13(3), 245-258.

Burke, Raymond R. (1996), "Virtual Shopping: Breakthrough in Marketing Research,” Harvard Business Review, Mar-Apr, 120-131.

Burke, Raymond R. (2005), “The Third Wave of Marketing Intelligence,” in Manfred Drafft and Murali Mantrala (Eds.): Retailing in the $21^{\text {st }}$ Century: Current and Future Trends, Springer, 113-125.

Camerer, Colin, and Teck-Hua Ho (1999), "Experience-weighted Attraction Learning in Normal Form Games,” Econometrica, 67(4), 827-874.

Catledge, Lara D. and James E. Pitkow (1995), "Characterizing Browsing Strategies in the World-Wide Web,” Computer Networks and ISDN Systems, 27 (6), 1065-1073.

Chandon, Pierre, J. Wesley Hutchison, and Scott H. Young (2001), "Measuring the Value of Point-of-Purchase Marketing with Commercial Eye-Tracking Data," Working Paper.

Chi, Ed H., Peter Pirolli, Kim Chen, and James Pitkow (2001), "Using Information Scent to Model User Information Needs and Actions on the Web,” SIGCHI’01, Seattle, WA.

Chintagunta, Pradeep K. (1993), "Investigating Purchase Incidence, Brand Choice and Purchase Quantity Decisions of Households,” Marketing Science, 12(2), 184-208. 
Chopard, Bastien, Pascal Luthi and Pierre-Antoine Queloz (1996), "Cellular Automata Model of car traffic in two-dimensional street networks,” Journal of Physics A, 29, 2325-2336.

Cormen, Thomas H., Charles E. Leiserson, Ronald L. Rivest, and Clifford Stein (2001), Introduction to Algorithms, $2^{\text {nd }}$ Ed. MIT Press.

Crist, Thomas O., David S. Guertin, John A. Wiens, and Bruce T. Milne (1992), “Animal Movement in Heterogeneous Landscapes: An Experiment with Eleodes Beetles in Shortgrass Prairie,” Functional Ecology, 6 (5), 536-544.

Day, Rong-Fuh, Gary C. W. Shyi, and Jyun-Cheng Wang (2006), "The Effect of Flash Banners on Multiattribute Decision Making: Distractor or Source of Arousal?” Psychology \& Marketing, 23(5), 369-382.

Dellaert, Benedict G., Theo A. Arentze, Michel Bierlaire, Aloy W.J. Borgers, and Harry J.P. Timmermans (1998), "Investigating Consumers' Tendency to Combine Multiple Shopping Purposes and Destinations,” Journal of Marketing Research, 35 (2), 177-188.

Du, Rex Y. and Wagner A. Kamakura (2006), "Household Life Cycles and Lifestyles in the United States,” Journal of Marketing Research, 43, 121-132.

Duchovski, Andrew T. (2002), “A Breadth-First Survey of Eye Tracking Applications,” Behavior Research Methods, Instruments, \& Computers, 34(4), 455-470.

Eirinaki, M., M. Vazirgiannis, and D. Kapogiannis (2005), "Web Path Recommendations based on Page Ranking and Markov Models," Proceedings of the $7^{\text {th }}$ annual ACM international workshop, 2-9, New York, NY.

Farley, John U. and L. Winston Ring (1966), "A Stochastic Model of Supermarket Traffic Flow,” Operations Research, July-Aug, 555-567.

Fox, Richard J., Dean M. Krugman, James E. Fletcher, and Paul M. Fischer (1998), "Adolescents' Attention to Beer and Cigarette Print Ads and Associated Product Warnings," Journal of Advertising Research, 27(3), 57-68.

Gogoi, Pallari (2005), “Retailing, the High-Tech Way,” BusinessWeek Online, July 6, Special Report: Retailing's New Tech.

Goldberg, Joseph H., Mark J. Stimson, Marion Lewenstein, Neil Scott, Anna M. Wichansky (2002), "Eye Tracking in Web Search Tasks: Design Implications," Proceedings of the symposium on Eye Tracking research \& applications, ACM Press, 51-58.

Guadagni P. M. \& J. D. C. Little (1983), "A Logit Model of Brand Choice Calibrated on Scanner Data,” Marketing Science, 2(3), 203-238. 
Harrell, Gilbert D., and Michael D. Hutt (1976), "Buyer Behavior Under Conditions of Crowding: An Initial Framework,” Advances in Consumer Research, Vol.3.

Harrell, Gilbert D., and James C. Anderson (1980), "Path Analysis of Buyer Behavior Under Conditions of Crowding,” Journal of Marketing Research, 17, 45-51.

Hauser, John R., Glen L. Urban, and Bruce D. Weinberg (1993), "How Consumers Allocate Their Time When Searching for Information,” Journal of Marketing Research, 15, 452466.

Helbing, Dirk, Joachim Keltsch and Peter Molnar (1997), "Modelling the Evolution of Human Trail Systems,” Nature, 388, 47-49.

Helbing, Dirk and Peter Molnar (1995), “Social Force Model for Pedestrian Dynamics,” Physics Review E, 51(5), 4282-4286.

Heller, Walter (1988), “Tracking Shoppers Through the Combination Store,” Progressive Grocer, 47-64.

Henderson, L. F. (1974), “On the Fluid Mechanics of Human Crowd Motion,” Transportation Research, 8, 509-515.

Hoffman, Donna L. and Thomas P. Novak (1996), “Marketing in Hypermedia ComputerMediated Environments: Conceptual Foundations,” Journal of Marketing, 60, 50-68.

Hui, Sam K., Peter S. Fader, and Eric T. Bradlow (2007), “Traveling Salesman Goes Shopping: The Systematic Deviations of Grocery Paths,” Working Paper.

Hui, Sam K., Eric T. Bradlow, and Peter S. Fader (2007), “An Integrated Model of Grocery Store Shopping Path and Purchase Behavior,” Working Paper.

Janiszewski, Chris (1993), “Preattentive Mere Exposure Effects,” Journal of Consumer Research, 20(3), 376-392.

Janiszewski, Chris (1998), “The Influence of Display Characteristics on Visual Exploratory Search Behavior,” Journal of Consumer Research, 25 (3), 290-301.

Jeanson, Raphael, Stephane Blanco, Richard Fournier, Jean-Louis Deneubourg, Vincent Fourcassie, and Guy Theraulaz (2003), “A Model of Animal Movements in a Bounded Space,” Journal of Theoretical Biology, 225, 443-451.

Kalawsky, Roy S. (1993), The Science of Virtual Reality and Virtual Environments: A Technical, Scientific, and Engineering Reference on Virtual Environments, Addison-Wesley.

Kamakura, Wagner A., and Gary J. Russell (1989), “A Probabilistic Choice Model for Market Segmentation and Elasticity Structure,” Journal of Marketing Research, 26(4), 379-390. 
Kim, Chang-Jin and Charles R. Nelson (1999), State-space Models with Regime Switching: Classical and Gibbs-Sampling Approaches with Applications. MIT Press.

Kim, Dong-Ho, Vijayalakshimi Atluri, Michael Bieber, Nabil Adam, and Yelena Yesha (2004), "A Clickstream-based Collaborative Filtering Personalization Model: Towards a Better Performance," Proceedings on the $6^{\text {th }}$ Annual ACM International Workshop on Web Information and Data Management, 88-95.

Krugman, Dean M., Richard J. Fox, James E. Fletcher, Paul M. Fischer, and Tina H. Rojas (1994), "Do Adolescents Attend to Warnings in Cigarette Advertising? An Eye-Tracking Approach,” Journal of Advertising Research, 39-52.

Larson, Jeffrey S., Eric T. Bradlow and Peter S. Fader (2005), “An Exploratory Look at Supermarket Shopping Paths,” International Journal of Research in Marketing, 22 (4), 395-414.

Lawler, Eugene L. (1985), The Traveling Salesman Problem: A Guided Tour of Combinatorial Optimization. Wiley.

Lee, Leonard, and Dan Ariely (2006), "Shopping Goals, Goal Concerteness, and Conditional Promotions,” Journal of Consumer Research, 33, 60-70.

Li, Hua-Fu, Suh-Yin Lee, and Man-Kwan Shan (2004), “On Mining Webclick Streams for Path Traversal Patterns,” Proceedings of the WWW2004.

Lohse, Gerald L. (1997), “Consumer Eye Movement Patterns on Yellow Pages Advertising,” Journal of Advertising, 16(1), 61-73.

Lurie, Nicholas, and Charlotte H. Mason (2007), "Visual Representation: Implications for Decision Making,” Journal of Marketing, 71, 160-177.

Mobasher, Bamshad, Honghua Dai, Tao Lou, Miki Nakagawa (2001), "Effective Personalization Based on Association Rule Discovery from Web Usage Data," Proceedings of the $3^{\text {rd }}$ ACM Workshop on Web Information and Data Management.

Moe, Wendy W. (2003), "Buying, Searching, or Browsing: Differentiating Between Online Shoppers Using In-Store Navigational Clickstream,” Journal of Consumer Psychology, 13, 29-39.

Moe, Wendy W., Hugh Chipman, Ed George, and Robert McCulloch (2002), “A Bayesian Treed Model of Online Purchasing Behavior Using In-Store Navigational Clickstream,” Working Paper.

Moe, Wendy W. and Peter S. Fader (2004), "Dynamic Conversion Behavior at E-Commerce Sites,” Management Science, 50(3), 326-335. 
Montgomery, Alan L., Shibo Li, Kannan Srinivasan, and John C. Liechty (2004), "Modeling Online Browsing and Path Analysis Using Clickstream Data," Marketing Science, 23(4), 579-595.

Moorman, Christine, Kristin Diehl, David Brinberg, and Blair Kidwell (2004), "Subjective Knowledge, Search Locations, and Consumer Choice,” Journal of Consumer Research, 31, 673-680.

Musalem, Andres, Eric T. Bradlow, and Jagmohan S. Raju (2006a), "Bayesian Estimation of Random-Coefficients Choice Models using Aggregate Data,” Journal of Applied Econometrics, to appear.

Musalem, Andres, Eric. T Bradlow, and Jagmohan S. Raju (2006b), "Who's got the coupon? Estimating Consumer Preferences and Coupon Usage from Aggregate Information”, Wharton School of the University of Pennsylvania working paper.

Otnes, Cele, and Mary Ann McGrath (2001), "Perceptions and Realities of Male Shopping Behavior,” Journal of Retailing, 77, 111-137.

Park, Young-Hoon, and Eric T. Bradlow (2005), “An Integrated Model for Bidding Behavior in Internet Auctions: Whether, Who, When, and How Much," Journal of Marketing Research, 42(4), 470-482.

Park, Young-Hoon, and Pater S. Fader (2004), "Modeling Browsing Behavior at Multiple Websites,” Marketing Science, 23, 280-303.

Payne, John W., James R. Bettman, and Eric J. Johnson (1988), “Adaptive Strategy Selection in Decision Making,” Journal of Experimental Psychology, 14(3), 534-552.

Pereira, Joe (2005), “Spying on the Sales Floor,” The Wall Street Journal, December 21, B1.

Pieters, Rik G.M., Edward Rosbergen, and Michel Hartog (1996), "Visual Attention to Advertising: The Impact of Motivation and Repetition," Advances in Consumer Research, 23, 242-248.

Pieters, Rik G.M., Edward Rosbergen, and Michel Wedel (1999), "Visual Attention to Repeated Print Advertising: A Test of Scanpath Theory,” Journal of Marketing Research, 18 (Nov), 424-438.

Pieters, Rik G.M., and Luk Warlop (1999), "Visual Attention During Brand Choice: The Impact of Time Pressure and Task Motivation,” International Journal of Research in Marketing, 16(1), 1-16. 
Pieters, Rik G.M., Luk Warlop, and Michel Wedel (2002), "Breaking Through the Clutter: Benefits of Advertising Originality and Familiarity for Brand Attention and Memory," Management Science, 48(6), 765-781.

Pieters, Rik G.M., and Michel Wedel (2004), "Attention Capture and Transfer in Advertisting: Brand, Pictorial, and Text-Size Effects,” Journal of Marketing, 68 (Apr), 36-50.

Polovina, Jeffrey J., Donald R. Kobayashi, Denise M. Parker, Michael P. Seki, and George H. Balazs (2000), "Turtles on the Edge: Movement of Loggerhead Turtles (Caretta caretta) along Oceanic Fronts, Spanning Longline Fishing Grounds in the Central North Pacific, 1997-1998,” Fisheries Oceanography, 9, 71-82.

Preisler Haiganoush K., Alan A. Ager, Bruce K. Johnson, and John G. Kie (2004), "Modeling Animal Movements using Stochastic Differential Equations," Environmetrics, 15, 643657.

Rabiner, Lawrence R. (1989), "A Tutorial on Hidden Markov Models and Selected Applications in Speech Recognition,” Proceedings of the IEEE, 777 (2), 257-286.

Ramsay, Jim, and Bernard W. Silverman (2005), Functional Data Analysis. Springer.

Rayner, Keith (1998), “Eye Movements in Reading and Information Processing: 20 Years of Research,” Psychological Bulletin, 124(3), 372-422.

Rayner, Keith, Caren M. Rotello, Andrew J. Stewart, Jessica Keir, and Susan A. Duffy (2001), "Integrating Text and Pictorial Information: Eye Movements When Looking at Print Advertisement,” Journal of Experimental Psychology: Applied, 7(3), 219-226.

Reynolds, Craig W. (1987), "Flocks, Herds and Schools: A Distributed Behavioral Model,” Computer Graphics, 21(4), 25-34.

Robertson, S. E. (1977), “Theories and Models in Information Retrieval,” Journal of Documentation, 33 (2), 126-148.

Rosbergen, Edward, Rik Pieters, Michel Wedel (1997), "Visual Attention to Advertising: A Segment-Level Analysis,” Journal of Consumer Research, 24(3), 305-314.

Rossi, Peter E., Greg Allenby, and Rob McCulloch (2006), Bayesian Statistics and Marketing. John Wiley and Sons.

Rudin, Walter (1976), Principles of Mathematical Analysis, $3^{\text {rd }}$ Edition, McGraw-Hill.

Sen, Sankar, and Eric J. Johnson (1997), "Mere-Possession Effects without Possession in Consumer Choice,” Journal of Consumer Research, 24(1), 105-117. 
Shahabi, Cyrus, Amir M. Zarkesh, Jafar Adibi , and Vishal Shah (1997), "Knowledge Discovery from Users Web-Page Navigation," Proceedings of IEEE Researcher Issues in Data Engineering.

Sismeiro, Catarina and Randolph E. Bucklin (2004), "Modeling Purchase Behavior at an ECommerce Web Site: A Task Completion Approach,” Journal of Marketing Research, 16, 306-323.

Song, Inseong, and Pradeep K. Chintagunta (2003), “A Micromodel of New Product Adoption with Heterogeneous and Forward-Looking Consumers: Application to the Digital Camera Category," Quantitative Marketing and Economics, 1(4), 371-407.

Sorensen, Herb (2003), “The Science of Shopping,” Marketing Research, 15(3), 30-35.

Stewart, Andrew J., Martin J. Pickering, and Patrick Sturt (2004), "Using Eye Movements during Reading as an Implicit Measure of the Acceptability of Brand Extensions," Applied Cognitive Psychology, 18, 697-709.

Sun, Baohong, Scott A. Neslin, and Kannan Srinivasan (2003), "Measuring the Impact of Promotions on Brand Switching when Consumers are Forward-Looking," Journal of Marketing Research, 40(Nov), 389-405.

Teknomo, Kardi, Yasushi Takeyama, and Hajime Inamura (2000), "Determination of Pedestrian Flow Performance Based on Video Tracking and Microscopic Simulations,” Proceedings of Infrastructure Planning Conference, 23 (1), 639-642.

Theusinger, Christiane and Klaus-Peter Huber (2000), "Analyzing the Footsteps of your Customers,” Proceedings of WebKDD 2000.

Thill, Jean-Claude, and Isabelle Thomas (1987), “Toward Conceptualizing Trip-Chaining Behavior: A Review,” Geographical Analysis, 19 (1), 1-17.

Thrun, Sebastian, Wolfram Burgard, and Dieter Fox (2005), Probabilistic Robotics, MIT Press.

Underhill, Paco (2000), How we buy: The Science of Shopping. Simon \& Schuster.

Underhill, Paco (2004), Call of the Mall: The Geography of Shopping. Simon \& Schuster.

Urban, Glen L., John R. Hauser, William J. Qualls, Bruce D. Weinberg, Jonathan D. Bohlmann, and Roberta A. Chicos (1997), "Information Acceleration: Validation and Lessons from the Field,” Journal of Marketing Research, 34(1), 143-153.

Vrechopoulos, Adam P., Robert M. O’Keefe, Georgios I. Doukidis, and George J. Siomkos (2004), "Virtual Store Layout: An Experimental Comparison in the Context of Grocery Retail,” Journal of Retailing, 80, 13-22. 
Wainer, Howard (2004), Graphic Discovery: A Trout in the Milk and Other Visual Adventures. Princeton University Press.

Zienkiewicz, Olgierd C., Robert L. Taylor and J. Z. Zhu (2005), The Finite Element Method: Its Basis and Fundamentals. Oxford: Elsevier Butterworth-Heinemann. 


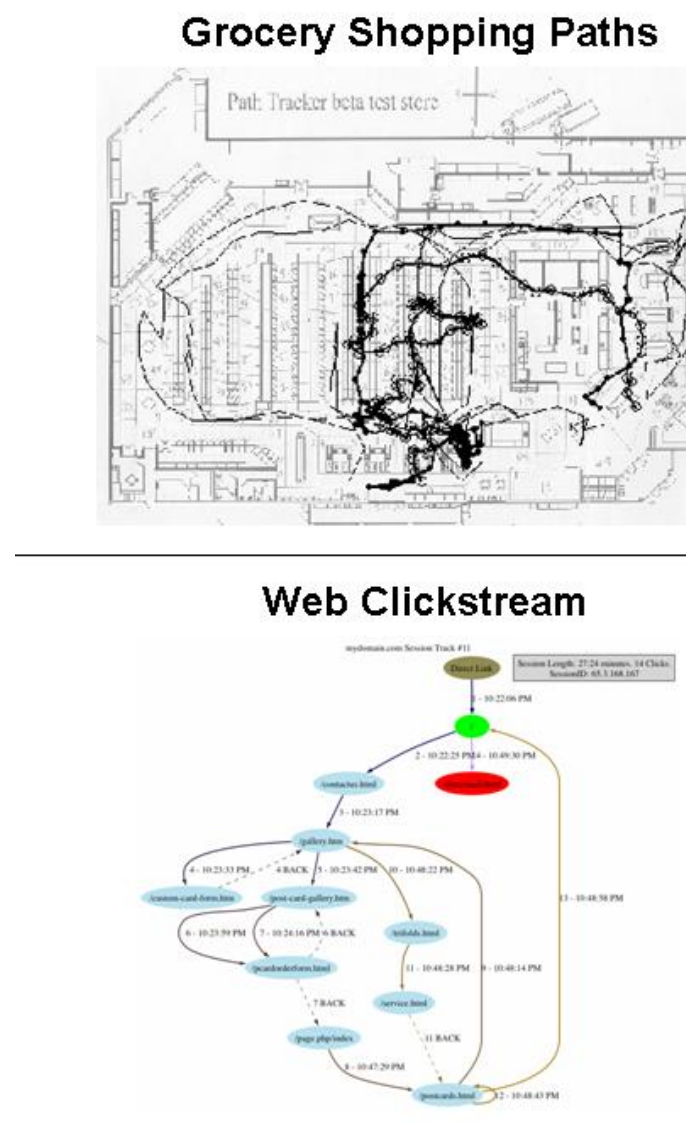

\section{Eye Tracking}

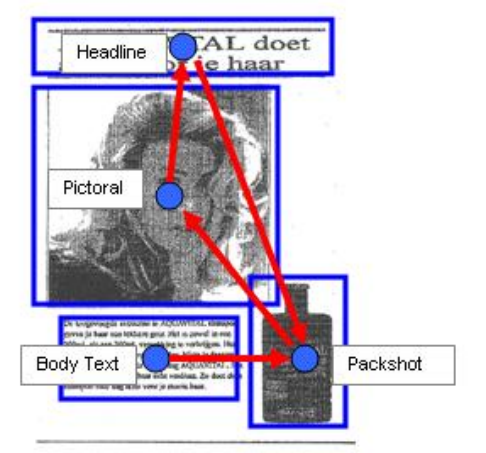

Physical movement

Figure 1. Four examples of path data 

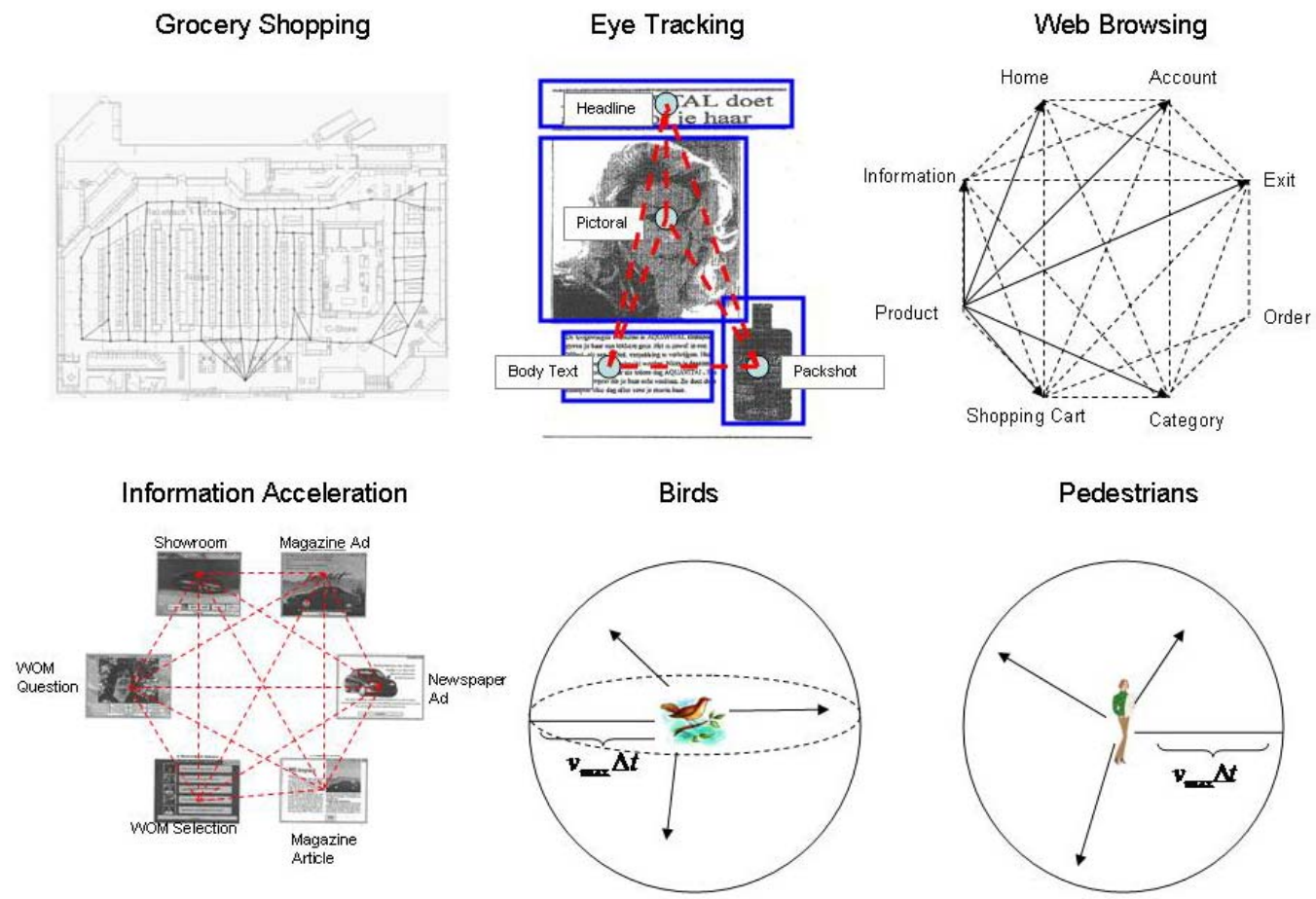

Figure 2. Space and Allowable Movements
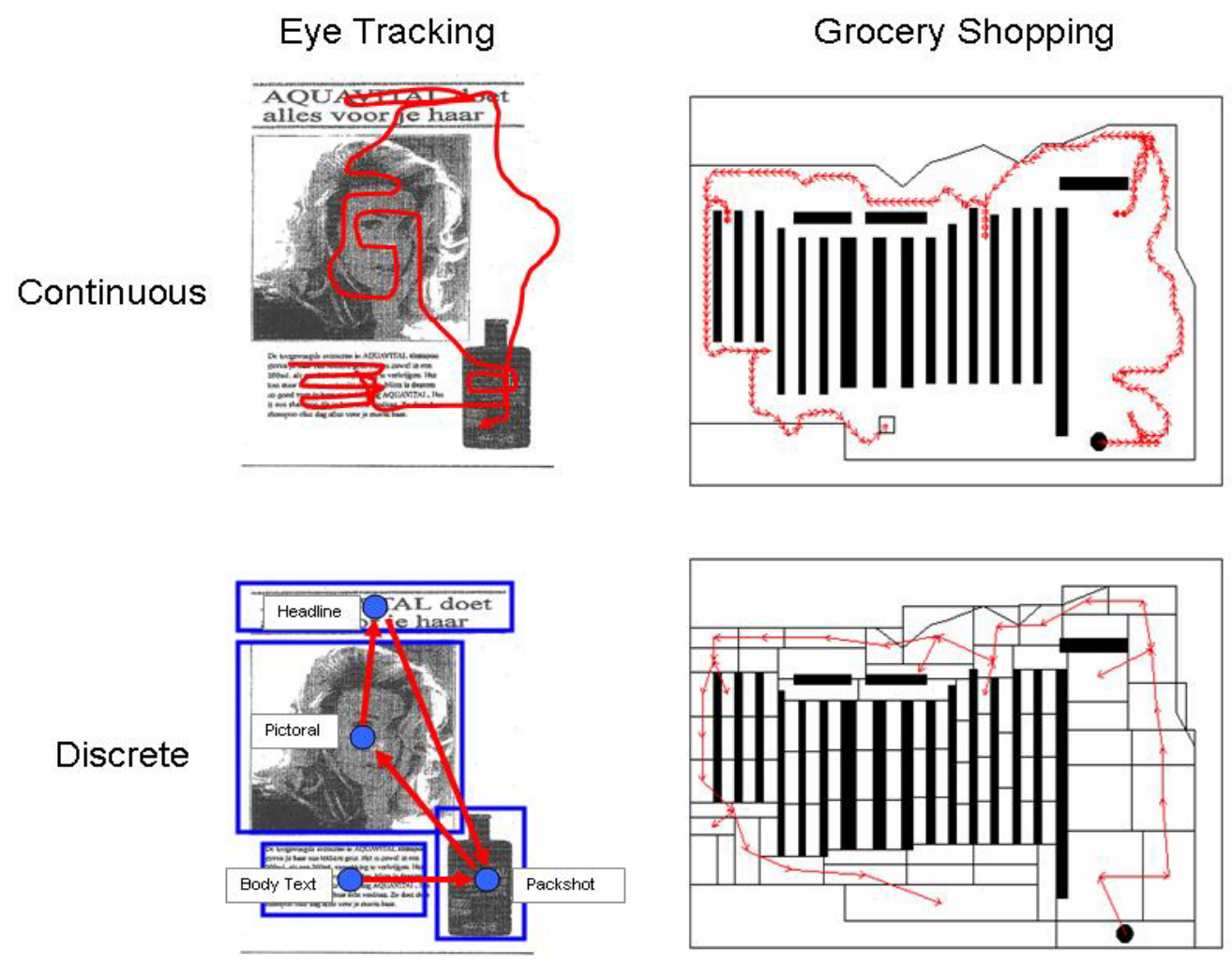

Figure 3. Discrete vs. continuous specification of eye-tracking and grocery shopping. 